\title{
The evaluation of Suchana, a large-scale development program to prevent chronic undernutrition in north-eastern Bangladesh: A large scale nutrition program design
}

Nuzhat Choudhury ( $\square$ nuzhat@icddrb.org )

International Centre for Diarrhoeal Disease Research https://orcid.org/0000-0001-8345-5278

Mohammad Jyoti Raihan

International Centre for Diarrhoeal Disease Research Bangladesh

S M Tanvir Ahmed

Save the Children Bangladesh

Kazi Eliza Islam

Save the Children Bangladesh

Vanessa Self

Save the Children

Shahed Rahman

Save the Children Bangladesh

Lilly Schofield

Save the Children

Andrew Hall

Save the Children

Tahmeed Ahmed

International Centre for Diarrhoeal Disease Research Bangladesh

Research article

Keywords: Randomized controlled trial, Stepped wedge design, Nutritional program evaluation, malnutrition

Posted Date: March 2nd, 2020

DOI: https://doi.org/10.21203/rs.3.rs-15632/v1

License: (9) (1) This work is licensed under a Creative Commons Attribution 4.0 International License.

Read Full License 


\section{Abstract}

\section{Background}

Evidence of the impact of community-based nutrition programs is uncommon for two main reasons: the lack of untreated controls, and implementation does not account for the evaluation design. Suchana is a large-scale program to prevent malnutrition in children in Sylhet Division, Bangladesh by improving the livelihoods and nutrition knowledge of very poor households.

\section{Methods}

Suchana is being implemented in 157 unions, the smallest administrative unit of government, in two districts of Sylhet. Suchana will deliver a package of interventions to poor people in about 40 randomly selected new unions annually over four years, until all are covered. All beneficiaries will receive the normal government nutrition services. For evaluation purposes the last 40 unions will act as a control for the first 40 intervention unions. The remaining unions will receive the program but will not take part in the evaluation. A baseline survey was conducted in both intervention and control unions; it will be repeated after three years to estimate the impact on the prevalence of stunted children and other indicators.

\section{Results}

This stepped wedge design has several advantages for both implementation and evaluation, as well as some disadvantages. The units of delivery are randomized, which controls for other influences on outcomes; the program supports government service delivery systems, so it is replicable and scalable; and the program can be improved over time as lessons are learned. The main disadvantages are the difficulty of estimating the impact of each component of the program, and the geographical distribution of unions, which increases program delivery costs. Stepped implementation allows a cluster randomized trial to be achieved within a large-scale poverty alleviation program.

\section{Conclusions}

A stepped wedge design for implementation was used which is particularly suited to evaluations of service delivery. The design allows the intervention to be phased-in and scaled-up over a period of time. The phased or 'stepped' process of implementation enables a cluster randomized controlled trial to be achieved without having a permanently untreated control group. However, the design does not allow to assess the impact of each individual component of intervention.

\section{Background}

In 2015, the World Health Assembly set a target to reduce by $40 \%$ the number of stunted children worldwide by 2025 (1). The prevalence of childhood stunting in Bangladesh is coming down by about $1.5 \%$ per year (2) and the country is almost on target, but the prevalence shows substantial variation around the country. In the last Bangladesh Demographic and Health Survey $49.6 \%$ of children less than 5 
years of age in Sylhet Division were stunted compared with $28.1 \%$ in Khulna Division, a difference of over 20 percentage points (2). Because of concern for this disparity, Save the Children (SC) designed and sought funding in partnership with other agencies for Suchana, a 7-year program that aims to prevent chronic malnutrition by delivering a package of nutrition-specific through government system and nutrition-sensitive development interventions by international and local non-government organizations (NGOs) to up to 250,000 very poor households in two districts of Sylhet Division.

The aim of the Suchana program beyond improving the nutrition and growth of children in the targets households is for successful elements to be replicated, scaled up and sustained while informing policies and other programs. The program has itself being built on operational learning from other large projects such as Stimulating Household Improvements Resulting in Economic Empowerment (shiree) (3) and The First Most Critical Day's Program (MCDP) in Bangladesh and also the vast amount of analysis such as Household Economy Analysis, The Cost of the Diet analysis, formative research, political economy analysis and social protection mapping informed the design. To achieve this effectively, robust evidence of the impact that is attributable to the interventions is required (4). This requires a counterfactual, so the program needs to be designed in a way that allows a concurrent control group to be achieved in the same geographic area at the same time with similar population characteristics. This challenge is one of the main reasons for the lack of robust evidence of the impact of programs on children's nutritional status (5). There is also a need to collect data on indicators of nutrition that minimise recall errors and social desirability bias so as to prevent over reporting of good behaviour (6).

This paper describes the design of the evaluation of Suchana, focusing on how the requirement for a counterfactual was balanced with the needs of a humanitarian development program, and discusses the advantages and disadvantages of the design.

\section{Methods}

Suchana is a large scale program that is being implemented by a consortium of agencies inclusive of technical, implementing and research partners: WorldFish, Helen Keller International (HKI), (International Development Enterprises) iDE, Rangpur Dinajpur Rural Service (RDRS) Bangladesh, Friends in Village Development Bangladesh (FIVDB), Center for Natural Resource Studies (CNRS), icddr,b and led by Save the Children, in Sylhet Division of north-eastern Bangladesh (Figure 1). Suchana aims to enrol up to 250,000 poor and very poor households in the program with the primary intention of preventing chronic undernutrition and linear growth retardation among children under two years of age born into these households during the program.

The primary outcome indicator is the mean z-score of height-for-age from which the percentage of children who are stunted ( $z$-score $<-2$ ) is derived. The secondary indicators are mostly related to infant feeding: how long after delivery the child was first breastfed; whether the child was exclusively breastfed for the first 6 months of life; whether a child aged $>1 \mathrm{y}$ is still being breastfed; the age at which solid, semisolid, or soft foods were introduced; the diversity of the diet in the last $24 \mathrm{~h}$; the number of meals in 
the last $24 \mathrm{~h}$; whether a minimum acceptable diet was given; whether iron-rich or iron-fortified foods were given (7); and the haemoglobin concentration.

A calculation was done to estimate the sample size of children required, in three age groups: $0-5$ months, 6-11 months and 12-23 months. This age range was chosen to be able to assess the impact of interventions in the 1,000 days after conception, the period during which undernutrition typically has its highest effect on linear growth.

For children aged 0-5 months the calculation was based on the prevalence of exclusive breastfeeding (EBF) from an unpublished study in Sylhet district of $55.3 \%$, an intra-cluster correlation coefficient (ICC) of 0.0293, an assumed increase of EBF of $10 \%$ points, a power of $80 \%$ and a level of significance of 0.05 . The sample size estimated was 520 subjects per group so the minimum total sample size for the baseline survey was 1,040 and for the final survey was 1,560, to allow for disaggregation of data into groups which did and did not receive asset transfers.

For children aged 6-11 months the primary outcome indicator was optimal infant and young child feeding (IYCF) practices. Using the information from the same unpublished survey which estimated a prevalence of $12.6 \%$, an ICC of 0.0235 , an assumed increase of $7 \%$, and the same power and level of significance the sample size was estimated at 600 per arm, so 1,200 in total and 1,800 in the final survey, to allow for disaggregation,

For children aged 12-23 months the sample size calculation was based on the main derived outcome indicator, the percentage of stunted children. In order to detect a difference of $6 \%$ points between the intervention and the untreated control group in 40 unions in each group after 3 years, the sample size required was estimated to be 1,520 children in each group assuming a power of $80 \%, 5 \%$ precision, 40 clusters per group and an ICC of 0.01 . To ensure adequate statistical power due to refusals or data collection errors, the final sample size was taken to be 3,200 , which was increased by more than $50 \%$ to 5,000 , to allow for disaggregation by asset transfer group.

Simple randomization technique was used to allocate the unions to four different phases or clusters. A baseline survey was carried out among beneficiaries of Suchana in 40 Phase 1 unions (intervention) and among potential beneficiaries in 40 phase 4 unions (control) in 2017. The survey included children aged 0 to 23 months and their parents, plus 1,200 adolescent boys and 1,200 girls aged 15 to 19 years to assess their knowledge of health and nutrition, to assess their decision making power, and to estimate the proportion who are already married, though the sample of the adolescents were themselves unmarried. Systematic sampling technique was used to selected beneficiary or potential beneficiary households to be assessed using the Suchana beneficiary/potential beneficiary list as the sampling frame. The final survey will be carried out in 2020. This design is shown in Figure 2.

\section{Results}

\section{Suchana program elements:}


There are five main elements to the program: (1) improved nutrition governance that translates political commitments into practice; (2) an effective and inclusive government service delivery system that enhances access to, and demand for, nutrition-related services to include the promotion of government nutrition services for pregnant women, mothers, and young children; (3) economic empowerment of the very poor, particularly women and adolescent girls, so that they can overcome economic barriers to better nutrition; (4) improving the knowledge, skills and power of women and adolescents girls to enable them to challenge harmful cultural practices and gender norms including gender-based violence, early marriage and early pregnancy that can affect mother and child nutrition; and (5) robust evidence of the impact of the package of interventions to prevent chronic malnutrition which will inform the policies and practices of the government and other agents of change. Households will be eligible to participate if they contain an eligible beneficiary type, meet locally determined proxy indicators for poverty, and are not currently taking part in any other livelihood development activity.

\section{Design for Suchana program implementation and evaluation:}

To be able to estimate the attributable impact of the package of interventions provided by Suchana and to control for the effect of other programs and factors in the same geographical area at the same time, a stepped wedge design for implementation was proposed by SC initially. This design is particularly suited to evaluations of service delivery, typically through government units of delivery such as the sub-district (8). In this instance, it is the union, the lowest tier of government in Bangladesh. This design allows the intervention to be phased-in and scaled-up over a period of time, so that the beneficiaries in the last group of unions can act as an untreated control group until they join the program in the last phase, while the beneficiaries in the first phase of unions are exposed to receive the interventions over a period of time sufficient to lead to a difference in outcome measures. This period is three years for the beneficiaries of Suchana. Following a pre post design for evaluation, a baseline survey before the program begins will serve to check that the outcome measures are not significantly different in children in potential target households in the intervention and control unions, and the survey will be repeated after 3 years intervention, at the same time of year to control for seasonality, economic fluctuations, to assess whether the null hypothesis, of no difference between children in the intervention and control unions, can be rejected.

The validity of any conclusions requires that the units of delivery are randomly allocated to the groups or phases. The phased or 'stepped' process of implementation enables a cluster randomized controlled trial to be achieved without having a permanently untreated control group, as the last phase of unions will eventually join the program for 3 years. The approach is also preferable from an ethical point of view as every person involved in the research also benefits from the program. All households in all unions are eligible to receive nutrition-specific interventions provided through the Government's National Nutrition Service (NNS) throughout the program, but these are promoted strongly by the program in the intervention unions. The design allows other effects of Suchana to be assessed providing that there is a plausible 
causal pathway between the interventions and a sensitive and specific outcome variable that is expected to change, and providing that the sample size is sufficient.

The design does not allow the impact of each individual component of Suchana to be estimated, but it may be possible to assess if the degree of exposure or participation in different elements of the program is associated with an outcome, by an analysis of variance of indicators pertaining to participation. The indicators of exposure will include the monetary value of investments in livelihoods such as fish or poultry farming, the income generated by these and other livelihood activities, and participation in behaviour change activities, such as the number of sessions attended by the mother of a child. Data will also be collected on household conditions, assets and circumstances, as they may also modify the outcome of Suchana. Data were collected on: household characteristics and assets; access to land and water bodies; water, sanitation and environmental hygiene; household dietary diversity and food security; expenditure, savings and loans; coping strategies, participation in other programs and income generating activities; health; access to government facilities and services; women's reproductive history, knowledge and empowerment; men's knowledge on child feeding and pregnancy care; and the characteristics of adolescents; engagement with market actors and access to social protection.

\section{Union assignment:}

The basis of assessing the impact is that the only difference between intervention and control households is, on average, due to the interventions delivered and supported by Suchana. Two districts of Sylhet Division, Sylhet and Maulvibazar, were selected for the program. They were chosen because no other large-scale Food Security and Nutrition (mother and child nutrition) programs were being implemented and they were not substantially within the 'hoar' wetlands, where the climatic and geographic conditions would make implementation at this large scale and within a fixed timeframe difficult. All 157 unions within these two districts, excluding commercial tea gardens and a small number of urban areas, were randomly allocated into four phases in a lottery, which took place in the presence of program staff, government staff and local elected representatives and dignitaries. This level of transparency was critical for community and government acceptance and all the union representatives understood that all unions would receive the program at some point. Forty unions were randomly allocated to phase 1 and 40 unions to phase 4, to achieve equal numbers; the other 77 unions were randomly allocated to phases 2 and 3. Each phase of Suchana will last 3 years (36 months) so that, by the end of the third year, all but the last phase will have joined the program and the households in phase 1 will have exposure benefited from 3 years of interventions. This process provided the intervention and control unions for the evaluation. This design is shown in Figure 2. As it is expected that all unions will behave similarly and the clusters were randomized to avoid any bias, Phase 2 and 3 were not considered in the evaluation.

\section{Beneficiaries selection:}


Villages in each union were selected for the program based on their vulnerability to poverty and natural disasters such as lack of development programs, poverty, remoteness and risk of flooding. This was done by a process of discussions with local government officials, representatives, and experts, and field visits by Suchana staff. In each village field staff used a participatory approach including focus group discussions and individual interviews with local people, to identify the poor and very poor poorest households to be targeted in each community. The wealth ranking sessions usually took place in the household courtyard, which is a convenient location to congregate from all corners covering approximately 150-200 households in each village. Using a process of participatory rural appraisal, households were allocated to one of four community-defined wealth groups. Communities are typically self-contained within village clusters of approximately 150-200 households. If any villages contained more than 200 households, two wealth ranking sessions were conducted, each joined by half of the household heads of the respective villages. The households ranked in the two poorest wealth groups were physically verified then visited by program field staff to validate that they met the inclusion criteria to be selected for Suchana.

It is to be noted that since a household's as the ability to implement new or scaled-up livelihoods and homestead gardens would likely depend on their degree of poverty, the program provide assets to the poorest to ensure that all beneficiaries would be in a position to engage in livelihood activities and take equal advantage of the participate training offered. It is expected that the ratio of asset-transfer to nonasset transfer beneficiaries is to be 1:1.5.

The evaluation design and methods received approval from the Research Review Committee (RRC) and Ethical Review Committee (ERC), the two obligatory components of the institutional review board (IRB) of icddr,b. Written informed consent was obtained from the parents, while for adolescents, written informed consent/assent consent was obtained from their parent or guardian. Local elites were involved while selecting the vulnerable villages and household heads of all villages were involved during the participatory rural appraisal process for selecting beneficiary households.

\section{Discussion}

The stepped wedge design to implement Suchana allows a randomized cluster-controlled trial of a package of interventions that has been designed to improve livelihoods, provide income, inform and empower girls and women, and improve the nutritional status of their children in area of Bangladesh that is lagging behind the country in an important indicator of national development, the percentage of stunted children.

Some of the notable interventions globally delivered in clustered randomized stepped wedge manner includes, The Gambian Hepatitis Intervention Study (GHIS) which was delivered in three phases over a period of four years (9), Evaluation of the Mexican universal health insurance program where 74 matched cluster pairs were chosen to deliver intervention and to serve as control (10), The multi-structured depression management in nursing homes conducted in The Netherlands using 17 nursing homes where 
they started to receive interventions randomly in one of the total five dates (11) and the Enhanced PeriOperative Care for High-risk patients (EPOCH) trial-an intervention of service delivery, where the intervention was provided sequentially to 90 hospitals of two level clustering $(12,13)$. The stepped wedge design has a number of advantages, both for development agencies and technical evaluators, as well as some disadvantages.

A program cannot easily be delivered to beneficiaries in individual households and at the same time have similar neighbours as controls, as this could lead to contamination or resentment. It also does not test the government service delivery mechanism. The best option is to deliver the program through the smallest unit of government, which in Bangladesh is the union. The staff of the union can then deliver the interventions to all beneficiaries in their union, selected on the basis of need by the community themselves using participatory methods, and the population understands that all beneficiaries in that union have been chosen based on those criteria. The program and the evaluation use the smallest unit of service delivery by the government, so the processes developed can be replicated and scaled up, while the union also provides rational clusters for the evaluation. The design is consistent with normal government processes.

The design allows for a counterfactual while delivering a program in a phased approach that is eventually delivered to all communities. Governments are rarely able to implement a new program in all areas from the same start date, so a phased approach allows the program to be scaled up over time. The rationale for this is understood by program staff, which is why it needs to be integrated into to the design from the start of the planning process. More the evaluation of large-scale nutrition sensitive programs has not been often done and Suchana evaluation will be a unique contribution to this field.

This method of program delivery allows a counterfactual, which is provided by a control group of future beneficiaries in 40 unions. This allows changes that would have occurred anyway to be subtracted from the change in the beneficiaries in the intervention group, and thus estimate the impact that is attributable to the intervention. The prevalence of stunted children is generally going down in Bangladesh, but with the program it should accelerate to go down more. The measurement of a single group before and after the program, the typical method used to evaluate programs (14), would not allow this, so a counterfactual is needed.

There is no untreated control group in this design and no placebo is needed (or possible). Every union gets the program eventually, so there are no ethical issues due to withholding treatment. Randomization is crucial, as in all controlled trials, to distribute differences between the units of delivery in the intervention and control groups. As the smallest unit of delivery of services by the government is the union, this was identified as the unit of delivery of Suchana and the unit of randomization for the evaluation, so creating a cluster randomized controlled trial. The randomization process, by which the phases of involvement of unions in the program was decided, was done transparently, with representatives of the unions present as observers, so they could see that there is no bias in the selection process, which is sometimes the case in programs. 
All unions will receive the program for the same number of years but, as the program is delivered in annual phases, it can be changed for unions in phases 2, 3 and 4, as lessons are learned and improvements made. It is not a fixed intervention, unlike a normal randomized controlled trial of a standard treatment, even if the interventions delivered to households in phase 1 are sustained. This is good for the program, which can be developed based on learning and experience, while the evaluation of impact continues.

The rate of reduction in the prevalence of stunted children is quite slow, even without a program; to achieve a difference between children in intervention and control unions that is statistically significant takes time. Three years was estimated to be the minimum period needed to show a difference and this influenced the scale of the program, the number of unions necessary for a cluster randomized trial, and the total budget, both for the program and the evaluation.

The advantages and benefits of the proposed evaluation design led the donors to fund a separate evaluation both in terms of the evaluators, to ensure objective and independent data were collected, and to fund it separately, so that it did not come out of the funding for the main program.

Separate entity enhances credibility and strengthen the process. However, proper consultation was done at different stages of design.

There are disadvantages of a stepped wedge design. The randomization process led to unions in phase 1 that are distributed throughout two districts, which increases the travel time of project staff from their base office to program unions, increases the cost of training government staff if they have to travel outside their unions to a central training venue, and increases program support costs. This makes it hard to estimate the cost effectiveness of implementing the program, as it will be higher than it need have been, and it also increases evaluation costs for the same reasons. The most cost-efficient way to implement a program would be to involve unions in contiguous blocks close to the project office in the first phase, and then spread out across the two districts, also in blocks. The issue was addressed partially by stratifying unions by geographical location before randomization.

The randomization process increases the risks of contamination of people in control unions from people in intervention unions, especially of public health messages that are specific to the program. Due to the scale of the program in terms of the number of unions required to achieve a sufficient number for a stepped wedge design and for a controlled evaluation, the program is expensive. However, considering the costs per beneficiary, impact at household level, impact at systems level, and research the program is indeed cost-effective and arguably provides better value-for-money than a traditional delivery approach, which cannot yield the same magnitude of results. The number of clusters in an evaluation is as important as the sample size of subjects studied, in order to control for inter-cluster differences. The Suchana program will cost GBP 50 million over seven years while the evaluation will cost around GBP 1.5 million. This is a substantial investment by donors, but it could generate high quality evidence. It is not an appropriate or possible design for all evaluations of programs to improve government services. 
The design does not easily allow the separate impact of each element of a package of interventions to be estimated. Development programs rarely deliver a single, pure intervention, such as a drug, and typically contain several elements, some supporting each other, while others may be independent of each other but may still be nutrition-sensitive. The only way to assess whether an element contributes to the difference in outcomes is to try to measure indicators of participation in the program or the quantity of intervention received, if it can be measured, and factors that might influence uptake and utilization by households. The challenge for the evaluators is to identify the indicators with implementers and then make sure that they are recorded accurately throughout the program.

It is also a challenge to identify potential beneficiaries in control unions during the baseline survey who have the same characteristics as potential beneficiaries in the intervention unions, and then not provide interventions. The same criteria used by the implementers to identify beneficiaries need to be used by evaluators to identify subjects for study in all unions in both surveys. At the final survey this is easier as the subjects for study could be included in the program when it begins in phase 4 .

This is when a close link between evaluators and program managers is important. The challenges of implementing a large complex program led to delays which had to be accommodated by the evaluation agency, so flexibility was required to undertake the baseline survey. The evaluation instruments were grounded in the specific program theory of each component of the intervention, to capture and record access to Suchana interventions, as well as to measure and capture contextual factors among Suchana workers, mothers, households, and communities, all of which can influence the effectiveness of the interventions.

The main element of the program that is not evaluated by the stepped wedge design is the process of implementation. This would require a separate evaluation design and evaluation process, so that all elements of the process related evaluation are separate and independent from the current evaluation design.

The randomization of 157 unions in two Districts of northern Bangladesh into four phases has allowed an evaluation to be designed to estimate the impact of interventions intended to prevent chronic malnutrition in children during the first 1,000 days of children's lives. The timing of the evaluation is also important- unlike in most program where the impact evaluation is completed at the end and by the time the results are available the program has wrapped up Suchana will be able to actively harness its impact evaluation for program learning and adjustment but also for advocacy to government and other partners for 3 years before the program finishes. The baseline evaluation started during November 2016 and ended in February 2017. This evaluation design is not suitable or feasible for all programs, but when it is finished in 2020, it may constitute one of the largest ever randomized controlled cluster trials of efforts to prevent stunting ever attempted.

\section{Conclusions}


A stepped wedge design for implementation was used which is particularly suited to evaluations of service delivery. The design allows the intervention to be phased-in and scaled-up over a period of time. The phased or 'stepped' process of implementation enables a cluster randomized controlled trial to be achieved without having a permanently untreated control group. However, the design does not allow to assess the impact of each individual component of intervention.

\section{List Of Abbreviations}

ICC: intra-cluster correlation coefficient

EBF: exclusive breastfeeding

\section{Declarations}

Ethics approval and consent to participate: The study was approved by the Research Review Committee (RRC) and Ethical Review Committee (ERC), the two obligatory components of the institutional review board (IRB) of icddr,b. During data collection, informed written consent was taken from parents. As for the adolescents, written informed consent/assent consent was obtained from their parent or guardian. The respondents were informed about their voluntary participation and right to withdraw at any point of time during the interview.

Consent for publication: Not applicable.

Availability of data and materials: Data sharing is not applicable to this article as no datasets were generated or analysed for the current study.

Competing interests: The first author is an associate editor of BMC Public Health. Otherwise, there is no conflict of interest.

Funding: The Suchana program and evaluation are funded by the United Kingdom Department for International Development (UKAid) and the European Union. The funders have no direct involvement with the implementation or evaluation of the Suchana program.

Authors' contributions: TA, AH, VS, and LS conceptualized the project. NC, MJR, KEI, and SMTA drafted the protocol. NC, MJR, AH, SMTA, SR, and TA drafted the manuscript. NC, MJR, SMTA, KEI, VS, SR, LS, AH and TA reviewed and edited the manuscript. All authors read and approved the final version of the manuscript.

Acknowledgements: The icddr,b is supported by the Government of the People's Republic of Bangladesh; the Department of Foreign Affairs, Trade and Development (DFATD), Canada; the Swedish International Development Cooperation Agency (SIDA); and UKAid. We would like to thank Save the Children International, Helen Keller International, World Fish, and International Development Enterprises, and their 
implementing partners Friends in Village Development Bangladesh, Rangpur Dinajpur Rural Services, and the Center for Natural Resource Studies, who helped to develop the data-collection tools.

\section{References}

1.World Health Organization. WHA global nutrition targets 2025: stunting policy brief.. Geneva; 2014.

2.NIPORT, Mitra and Associates, ICF International. Bangladesh Demographic and Health Survey 2014. Dhaka, Bangladesh, and Rockville, Maryland, USA: National Institute of Population Research and Training (NIPORT); 2016.

3.SHIREE. Stimulating Household Improvements Resulting in Economic Empowerment. 2016 [cited 2019. Available from: http://www.shiree.org/.

4.Menon $\mathrm{P}$, Rawat R, Ruel M. Bringing rigor to evaluations of large-scale programs to improve infant and young child feeding and nutrition: the evaluation designs for the Alive \& Thrive initiative. Food and nutrition bulletin. 2013;34(3_suppl2):S195-S211.

5.TANGO International Inc. Quantitative impact evaluation of the SHOUHARDO II Project in Bangladesh 2015.

6.Frongillo EA. Evaluation of programs to improve complementary feeding in infants and young children. Maternal \& child nutrition. 2017;13:e12436.

7.World Health Organization. Indicators for assessing infant and young child feeding practices: part 2: measurement. 2010.

8.Save the Children. Suchana: Ending the Cycle of Undernutrition in Bangladesh. Proposal Submission to Department for International Development, Bangladesh and the European Union Delegation, Bangladesh. 2015.

9. Hall A, Inskip H, Loik F, Day N, O'Connor G, Bosch X, et al. The Gambia hepatitis intervention study. Cancer Res. 1987;47(21):5782-7.

10.King G, Gakidou E, Imai K, Lakin J, Moore RT, Nall C, et al. Public policy for the poor? A randomised assessment of the Mexican universal health insurance programme. The lancet. 2009;373(9673):144754.

11.Hemming K, Haines TP, Chilton PJ, Girling AJ, Lilford RJ. The stepped wedge cluster randomised trial: rationale, design, analysis, and reporting. Bmj. 2015;350:h391.

12.Pearse R, Pedan C, Bion J, Faiz O, Holt P, Girling A. HS\&DR-12/5005/10. Enhanced Peri-Operative Care for High-risk patients (EPOCH) trial: a stepped wedge cluster randomised trial of a quality improvement intervention for patients undergoing emergency laparotomy [protocol]. 2013. 
13.Peden CJ, Stephens T, Martin G, Kahan BC, Thomson A, Rivett K, et al. Effectiveness of a national quality improvement programme to improve survival after emergency abdominal surgery (EPOCH): a stepped-wedge cluster-randomised trial. The Lancet. 2019.

14. Habicht J-P, Pelto GH, Lapp J. Methodologies to evaluate the impact of large scale nutrition programs. Washington, DC: World Bank. 2009.

\section{Figures}

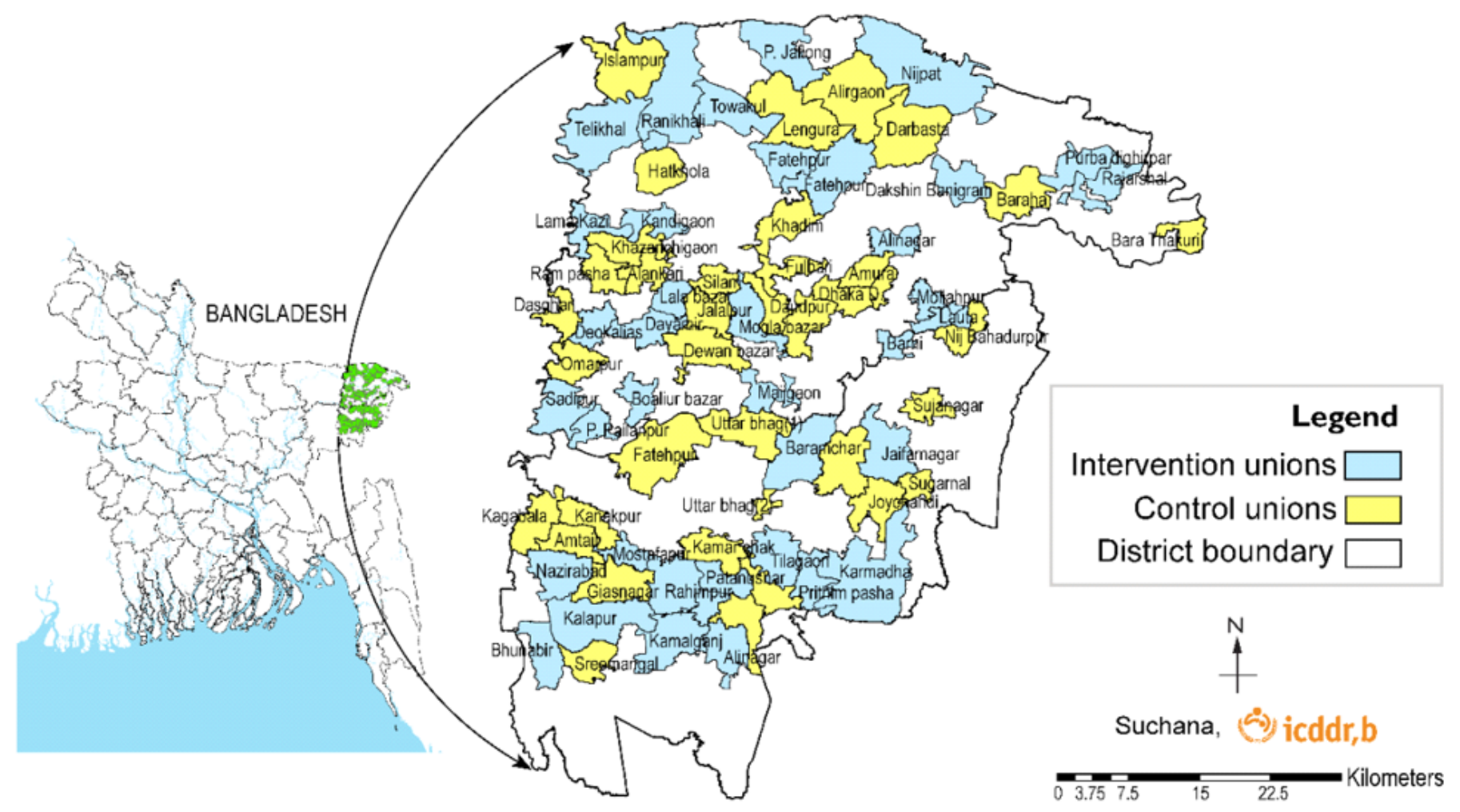

\section{Figure 1}

A map of the two districts in Sylhet Division showing the unions randomly selected for phase 1 of Suchana (intervention) and phase 4 (control). The figure was created by the authors. 


\begin{tabular}{|l|l|l|l|l|}
\hline & Year 1 & Year 2 & Year 3 & Year 4 \\
& & & & \\
\hline Suchana phase 4 (Control) & & & \multicolumn{2}{|c|}{} \\
\hline Suchana phase 3 & & & 39 & 39 \\
\hline Suchana phase 2 & & 38 & 38 & 38 \\
\hline Suchana phase 1 (Intervention) & 40 & 40 & 40 & \\
\hline Time & $\mathrm{T}_{0}$ & $\mathrm{~T}_{1}$ & $\mathrm{~T}_{2}$ & $\mathrm{~T}_{3}$ \\
\hline Surveys & $\mathrm{SB}$ & & & $\mathrm{SE}$ \\
\hline & & & & \\
\hline
\end{tabular}

\section{$\underline{\text { Key }}$}

\begin{tabular}{|l|l|}
\hline $\begin{array}{l}\text { SB } \\
=\end{array}$ & $\begin{array}{l}\text { Baseline survey, to establish baseline values and similarity of Phase 1 (P1) and Phase 4 } \\
(\mathrm{P} 4)\end{array}$ \\
\hline $\begin{array}{l}\text { SE } \\
=\end{array}$ & \begin{tabular}{l} 
Final survey, to assess attributable impact of intervention in P1 unions \\
\hline$\nearrow$
\end{tabular} \\
$=$
\end{tabular}

Figure 2

The Suchana evaluation diagram

\section{Supplementary Files}

This is a list of supplementary files associated with this preprint. Click to download.

- CONSORT2010Checklist.doc 